\title{
Observation of Entanglement between Two Light Beams Spanning an Octave in Optical Frequency
}

\author{
Nicolai B. Grosse, ${ }^{1}$ Syed Assad,,${ }^{1,2}$ Moritz Mehmet, ${ }^{3}$ Roman Schnabel, ${ }^{3}$ Thomas Symul, ${ }^{1}$ and Ping Koy Lam ${ }^{1}$ \\ ${ }^{1}$ Quantum Optics Group, Department of Physics, Faculty of Science, The Australian National University, ACT 0200, Australia \\ ${ }^{2}$ Department of Physics, National University of Singapore, Singapore 117542 \\ ${ }^{3}$ Max-Planck-Institut für Gravitationsphysik (Albert-Einstein-Institut), Leibniz Universität Hannover, \\ Callinstraße 38, 30167 Hannover, Germany \\ (Received 26 March 2008; published 18 June 2008)
}

\begin{abstract}
We have experimentally demonstrated how two beams of light separated by an octave in frequency can become entangled after their interaction in a $\chi^{(2)}$ nonlinear medium. The entangler was a nonlinear optical resonator that was strongly driven by coherent light at the fundamental and second-harmonic wavelengths. An interconversion between the fields created quantum correlations in the amplitude and phase quadratures, which were measured by two independent homodyne detectors. Analysis of the resulting correlation matrix revealed a wave function inseparability of $0.74(1)<1$, thereby satisfying the criterion of entanglement.
\end{abstract}

DOI: 10.1103/PhysRevLett.100.243601

Central to modern techniques in optical metrology has been the ability to make connections between light beams that span an octave in optical frequency. This development has realized the optical comb whose offset frequency can be directly linked to the SI definition of the second [1]. As such, spectroscopic measurements can have an absolute accuracy beyond one part in $10^{15}$ [2] and have enabled unprecedented testing of fundamental quantum mechanical effects [3]. Parallel to this has been progress in the area of quantum optics, where sources of nonclassical light have pushed the sensitivity of interferometric measurements beyond their associated classical bounds [4]. Recent achievements in the strength and stability of squeezed light sources suggest their imminent application to improving the sensitivity of ground-based gravitational wave detectors [5]. Correspondingly, a source of entangled light with beams that span an octave in optical frequency and which share quantum correlations, called harmonic entanglement, has the potential to be applied to the heterodyne stabilization of optical combs used in metrology [6].

The goal of producing harmonic entanglement has only recently been actively pursued by experimental groups worldwide. Milestones include demonstrations of twocolor discrete-variable entanglement [7] and continuousvariable entanglement from nondegenerate parametric processes [8]. The large spans offered by harmonic entanglement were predicted to occur in second-order nonlinear processes such as second-harmonic generation (SHG), above-threshold optical parametric oscillation (OPO), and optical parametric amplification (OPA) [9-11]. Although a quantum correlation in amplitude between the fundamental and second-harmonic fields of SHG has been observed [12], confirmation of entanglement also requires the detection of phase quadrature correlations of the (necessarily) bright light beams. Techniques have been used to rotate the phase quadrature onto the amplitude quadrature by using either an undercoupled optical reso-
PACS numbers: 42.50.Dv, 03.67.Bg, 42.50.Ex, 42.65.Yj

nator or an unequal arm length Mach-Zehnder interferometer [13]. Yet with the most recent results from nondegenerate OPO appearing limited by excess phase noise [14], a demonstration of harmonic entanglement has remained elusive.

In this Letter, we present observations of entanglement in continuous variables between two modes of light at 532 and $1064 \mathrm{~nm}$ spanning an octave. Our best results show a wave function inseparability of $0.74(1)<1$ between the reflected fundamental and second-harmonic fields of a degenerate OPA. We overcame the problem of excess phase noise by choosing the driving field parameters such that the noise effectively canceled. In addition, we demonstrated the technique of optical carrier rejection (OCR) using impedance-matched cavities, which enabled us to directly perform sideband homodyne detection on light beams that were originally an order of magnitude brighter than the local oscillator. From measurements of the amplitude and phase quadratures, we determined the correlation matrix elements and tested for entanglement. We exploited the flexibility of our experimental setup to smoothly drive the system from parametric amplification to deamplification. The results are in good agreement with a model of pump-depleted OPA.

The OPA process is described by an efficient phasedependent exchange of energy between a fundamental seed and second-harmonic pump field. Because of this process, the light fields reflected from an OPA can be entangled in their amplitude and phase quadratures. Our aim is to obtain expressions for these observables and apply the inseparability criterion to verify entanglement. We extend our previous model [11] to include a term that describes the effect of guided acoustic-wave Brillouin scattering (GAWBS). Consider a mode of light $a$ and its second-harmonic $b$ (wavelengths $\lambda_{a}=2 \lambda_{b}$ ), which interact via a nonlinearity of strength $\epsilon$ in a single mode cavity with total decay rates $\kappa_{a, b}$. The intracavity fields are 
coupled to the environment through a mirror $\kappa_{a 1, b 1}$ and more weakly via other loss mechanisms $\kappa_{a 2, b 2}$. The system is driven by two coherent fields $\alpha_{\text {in }}$ and $\beta_{\text {in }}$ and can be modeled by [15]

$$
\begin{gathered}
\dot{\hat{a}}=-\left(\kappa_{a}+i \delta w_{a}\right) \hat{a}+\epsilon \hat{a}^{\dagger} \hat{b}+\hat{A}_{\mathrm{in}}, \\
\dot{\hat{b}}=-\left(\kappa_{b}+i \delta w_{b}\right) \hat{b}-\frac{1}{2} \epsilon \hat{a}^{2}+\hat{B}_{\mathrm{in}},
\end{gathered}
$$

where input fields are denoted by $\hat{A}_{\text {in }}=\sqrt{2 \kappa_{a 1}} \alpha_{\text {in }}+$ $\sum \sqrt{2 \kappa_{a, j}} \delta \hat{A}_{j, \text { in }}, \hat{B}_{\text {in }}=\sqrt{2 \kappa_{b 1}} \beta_{\text {in }}+\sum \sqrt{2 \kappa_{b, j}} \delta \hat{B}_{j, \text { in }}$, with $j \in\{1,2\}, \quad \kappa_{a}=\kappa_{a 1}+\kappa_{a 2}$, and $\kappa_{b}=\kappa_{b 1}+\kappa_{b 2}$. The GAWBS noise is modeled by the detuning terms $\delta w_{a, b}=$ $\left(-2 \pi c / \lambda_{a, b}\right) \xi_{a, b} \delta P$, which are driven by the dimensionless noise term $\delta P$ having variance one but coupled via $\xi_{a, b}$; see [16].

We work in the Heisenberg picture where the annihilation and creation operators evolve, from which the amplitude and phase quadrature operators are constructed, $\hat{X}^{+}=\hat{a}^{\dagger}+\hat{a}$ and $\hat{X}^{-}=i\left(\hat{a}^{\dagger}-\hat{a}\right)$, respectively. For simplicity, we drop the hat notation henceforth. Operator linearization is used to obtain the fluctuations $\left(\delta X_{a}^{ \pm}\right.$and $\left.\delta X_{b}^{ \pm}\right)$centered around the classical steady-state solutions $(\alpha$ and $\beta$ ) [15]. Fourier transforming into the frequency domain allows one to solve for the driving fields in terms of the intracavity fields. This dependence is reversed when the equations are expressed in a matrix whose inverse is found:

$$
\left[\begin{array}{c}
\delta X_{a}^{+} \\
\delta X_{a}^{-} \\
\delta X_{b}^{+} \\
\delta X_{b}^{-} \\
\delta P
\end{array}\right]=\left[\begin{array}{ccccc}
A_{-} & B & C & D & F_{a} \\
B & A_{+} & -D & C & G_{a} \\
-C & D & E & 0 & F_{b} \\
-D & -C & 0 & E & G_{b} \\
0 & 0 & 0 & 0 & 1
\end{array}\right]^{-1}\left[\begin{array}{c}
\delta X_{A, \text { in }}^{+} \\
\delta X_{A, \text { in }}^{-} \\
\delta X_{B, \text { in }}^{+} \\
\delta X_{B, \text { in }} \\
\delta P^{\prime}
\end{array}\right],
$$

where $\left\{\delta X_{a}^{ \pm}, \delta X_{b}^{ \pm}\right\}$and $\left\{\delta X_{\bar{A}, \mathrm{in}}^{ \pm}, \delta X_{\bar{B}, \mathrm{in}}^{ \pm}\right\}$are the intracavity and accumulated input field quadratures, respectively; $A_{ \pm}=\kappa_{a}-i \Omega \pm \epsilon|\beta| \cos \theta_{\beta}, \quad B=-\epsilon|\beta| \sin \theta_{\beta}, \quad C=$ $-\epsilon|\alpha| \cos \theta_{\alpha}, \quad D=-\epsilon|\alpha| \sin \theta_{\alpha}, \quad E=\kappa_{b}-i \Omega, \quad F_{a, b}=$ $2 i|\alpha, \beta| \sin \theta_{\alpha, \beta}\left(-2 \pi c / \lambda_{a, b}\right) \xi_{a, b}, \quad G_{a, b}=2|\alpha, \beta| \times$ $\cos \theta_{\alpha, \beta}\left(-2 \pi c / \lambda_{a, b}\right) \xi_{a, b}$, with $\theta_{\alpha}=\arg (\alpha), \theta_{\beta}=\arg (\beta)$, and $\Omega$ is the sideband frequency. The fields reflected from the resonator can be directly obtained from: $\delta X_{A 1 \text {, ref }}^{ \pm}=$ $\sqrt{2 \kappa_{a 1}} \delta X_{a}^{ \pm}-\delta X_{A 1, \text { in }}^{ \pm} ; \quad \delta X_{B 1, \text { ref }}^{ \pm}=\sqrt{2 \kappa_{b 1}} \delta X_{b}^{ \pm}-\delta X_{B 1 \text {,in }}^{ \pm}$ [17]. Mode ${ }_{A 1 \text {,in }}$ was assigned a weak thermal state according to $\left\langle\left(\delta X_{A 1, \text { in }}^{ \pm}\right)^{2}\right\rangle=1+s\left|\alpha_{\text {in }}\right|^{2}$, with $s=10^{-3}$ per milliwatt, and similarly for mode ${ }_{B 1, \text { in }}$. All input states were treated as being uncorrelated.

The resulting bipartite Gaussian states are completely described by the correlation matrix of elements $C_{m n}^{k l}=\frac{1}{2} \times$ $\left\langle\delta X_{m}^{k} \delta X_{n}^{l}+\delta X_{n}^{l} \delta X_{m}^{k}\right\rangle$, given by

$$
M_{a b}=\left[\begin{array}{cccc}
C_{a a}^{++} & C_{a a}^{+-} & C_{a b}^{++} & C_{a b}^{+-} \\
C_{a a}^{-+} & C_{a a}^{--} & C_{a b}^{-+} & C_{a b}^{--} \\
C_{b a}^{++} & C_{b a}^{+-} & C_{b b}^{++} & C_{b b}^{+-} \\
C_{b a}^{-+} & C_{b a}^{--} & C_{b b}^{-+} & C_{b b}^{--}
\end{array}\right],
$$

where $\{k, l\} \in\{+,-\}$ and the reflected field notation has been simplified with $\{m, n\} \in\left\{A 1_{\text {ref }} \mapsto a, B 1_{\text {ref }} \mapsto b\right\}$. We use the quantity of inseparability $I$ as a measure of entanglement [18]. It is defined from $M_{a b}$ in the following way:

$$
\begin{gathered}
I=\left(C_{I}^{+}+C_{I}^{-}\right) /(2 k+2 / k), \\
C_{I}^{ \pm}=k C_{a a}^{ \pm \pm}+(1 / k) C_{b b}^{ \pm \pm}-2\left|C_{a b}^{ \pm \pm}\right|, \\
k^{ \pm}=\left[\left(C_{b b}^{ \pm \pm}-1\right) /\left(C_{a a}^{ \pm \pm}-1\right)\right]^{1 / 2} .
\end{gathered}
$$

The inseparability criterion $I<1$ is a necessary and sufficient condition for entanglement provided that the correlation matrix has been brought into standard form, which is obtained by applying local symplectic transformations $r_{a, b}$ to each mode separately ( $\delta X_{a}^{ \pm} \mapsto e^{ \pm r_{a}} \delta X_{a}^{ \pm}$, etc.) to minimize $I$ and satisfy $k^{+}=k^{-}=k$ [19]. $I$ takes values in the range $[0, \infty)$ with zero signifying perfect entanglement and unity the classical limit.

A map of inseparability as a function of seed and pump fields is plotted in Fig. 1. The entangled states produced by the OPA are biased, which means that the correlations of both quadratures are not of equal strength. These biased states, however, may still be optimally entangled $(I \rightarrow 0)$, in the ideal limit of a lossless system [20]. Except for the $\xi_{a, b}$ which were fitted to the phase quadrature measurements, all parameters used in our model were obtained by characterization of the experimental setup [21].

Figure 2 shows a schematic of the experiment. Generating and measuring harmonic entanglement demands the construction of an OPA optimized for the conflicting requirements of high escape efficiency and low threshold power. Only bright driving fields can yield strong entanglement, but the measurement of phase on bright fields is technically difficult. A Nd:YAG laser with an internal frequency doubler produced continuous-wave light at wavelengths $1064 \| 532 \mathrm{~nm}$, where " || " refers to parameters for each wavelength in that order. The light first needed spatial and temporal filtering via transmission

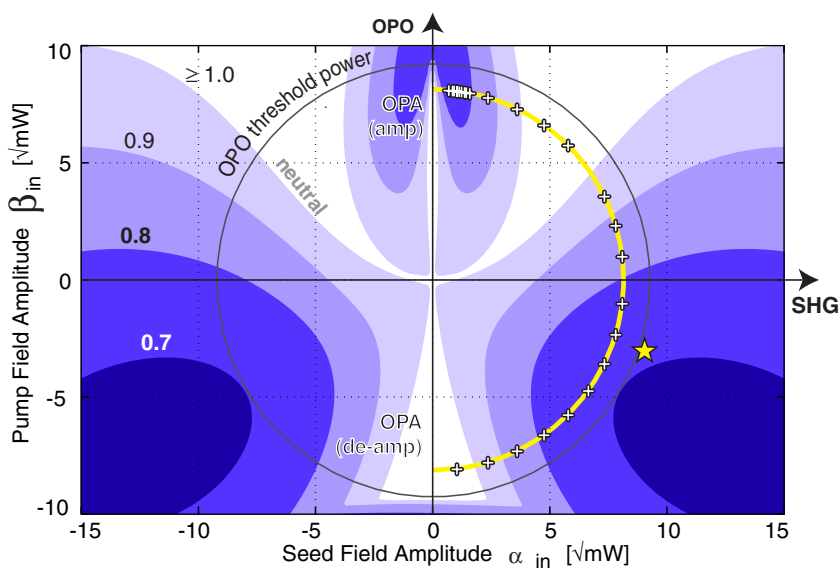

FIG. 1 (color online). Map of entanglement (inseparability) as a function of seed and pump driving fields as based on our OPA model. Darker shading indicates stronger entanglement. Symbols mark where regions have been experimentally probed. 


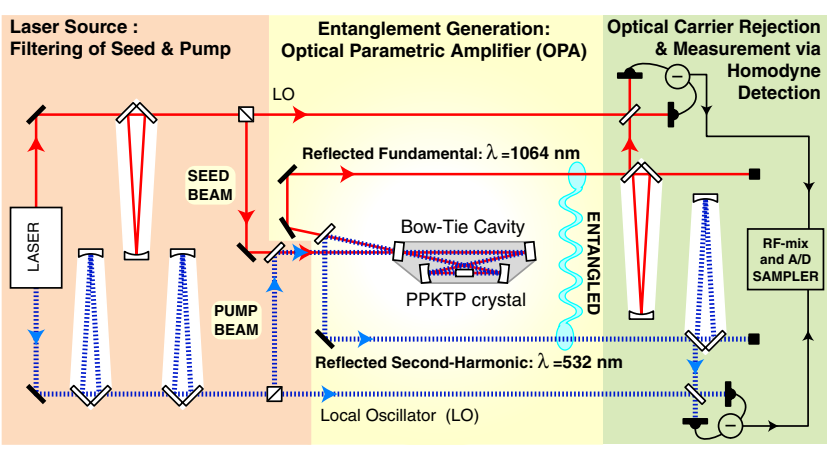

FIG. 2 (color online). Entanglement generation between the reflected fundamental and second-harmonic light of an OPA.

through optical cavities with combined linewidths of $0.4 \mathrm{MHz} \| 0.6 \mathrm{MHz}$. Both beams were near shot-noiselimited at $7.8 \mathrm{MHz}$ to within $0.1 \%$ per milliwatt of power. A fraction of the light was diverted for the local oscillator beams (LO), before preparing the seed and pump beams with a set of amplitude and phase modulations at frequencies $>20 \mathrm{MHz}$ that were used for servo control.

The entangler itself consisted of a PPKTP crystal in a bow-tie cavity with finesses of $57 \| 8.8$ and linewidths of $18 \| 120 \mathrm{MHz}$. The mirror reflectivities and intracavity losses gave escape efficiencies of $92 \| 86 \%$. The cavity length was controlled to resonate with the seed field. The pump field was made to coresonate by adjusting the crystal temperature. We obtained 98 || 99\% of cavity mode matching and achieved an optimized OPO threshold of $P_{\text {th }}=$ $85(5) \mathrm{mW}$. After interaction, the reflected beams (now entangled) were separated, and a $1 \%$ tap-off on each was detected. This enabled locking of the seed-pump relative phase so that the OPA could operate as either an amplifier or a deamplifier.

The vital step in detection was to remove the carrier light while preserving the sidebands. By using near impedancematched cavities with linewidths of $0.4 \| 1.0 \mathrm{MHz}$, it was possible to reject $21 \| 26 \mathrm{~dB}$ of carrier light and spatially mode-matched the OPA to 99.9(1) || 99.9(1)\%. Two homodyne detectors received the remaining reflected light which contained the sidebands, along with a small contribution of higher-order spatial modes originating from mode mismatch. Each homodyne detection setup had a pair of photodiodes. The estimated total quantum efficiency was 85(4) $\|$ 80(6)\%, and the shot-noise to darknoise clearance was $15 \mathrm{~dB}$. The LO phase was locked to either the phase or amplitude quadratures of the signal beam. Before taking entanglement readings, the locked quadrature angles were aligned to coincide with the maximum or minimum noise power. This guaranteed orthogonality of the quadratures being measured and allowed us to infer that the cross-quadrature correlations of a single color $\left(C_{a a}^{+-}\right.$, etc. $)$were zero. We have achieved a homodyne condition with signal to LO power of 1: $>30$, representing up to $26 \mathrm{~dB}$ filtering of the carrier field while preserving almost all of the sideband signal.
The homodyne detector photocurrents were independently mixed down at $7.8 \mathrm{MHz}$, before being sampled at $44.1 \mathrm{kHz}$ to acquire $2.6 \times 10^{5}$ points. The correlation matrix elements were evaluated from the paired measurements of the homodyned signal beams $\left(\delta X_{a}^{ \pm}\right.$and $\left.\delta X_{b}^{ \pm}\right)$ together with calibration traces. Corrections were made for the offsets incurred by dark noise and excess noise from higher-order spatial modes in the signal beam (which are artifacts of the OCR process and not the entangler itself). The entire experiment proved stable enough to allow us to complete a reliable measurement of the correlation matrix in more than $80 \%$ of all trials.

Results. -We drove the entangler across a range of parametric processes covering OPO, SHG, and OPA by fixing the total driving field power to $76 \%$ of $P_{\text {th }}$ and adjusting the balance of power between seed and pump. In Fig. 3, wave function inseparability is plotted as a function of the polar angle of Fig. 1, defined by $\phi=$ $\tan ^{-1}\left(\beta_{\text {in }} / \alpha_{\text {in }}\right)$. By moving away from pure OPO, entanglement was observed over a broad range of angles $(-0.41,+0.15) \pi$, which covered parametric deamplification through to SHG, and approached the neutral point (where net interconversion of fundamental and second harmonic is zero). The effect of pump enhancement in this region was prominent. Most of the fundamental field was converted into the second-harmonic field. We also found entanglement in the narrow range of $(+0.40,+0.47) \pi$ corresponding to parametric amplification with weak pump depletion. The entanglement observed in the pump-enhanced region was slightly better than in the pump-depleted region with $I=0.76(2)$ and $I=0.79(2)$, respectively. The dashed curve in Fig. 3 is a prediction based on a previous model [11], while the solid curve is from the extended model with GAWBS noise. The latter agrees well with the experimental results.

We observed the best harmonic entanglement in the parametric deamplification region of strong pump enhancement with powers at $81 \| 9 \mathrm{~mW}$ (see $\star$ in Fig. 1).

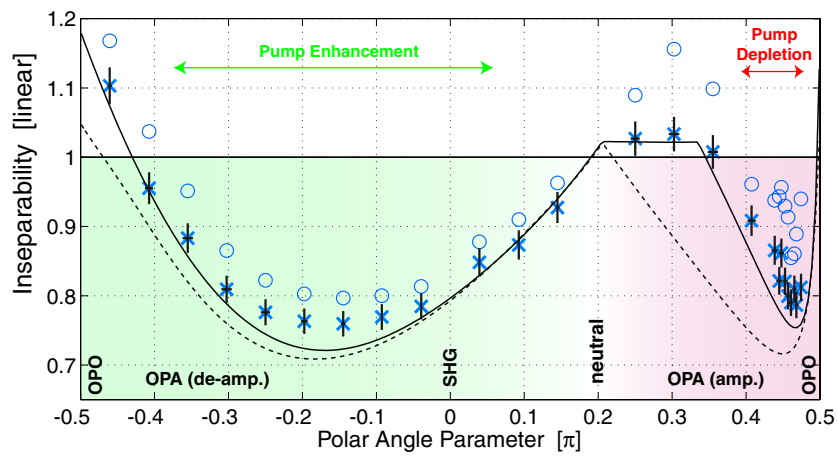

FIG. 3 (color online). Inseparability as a function of angle parameter with total input power held at $65 \mathrm{~mW}$. Entanglement is achieved for values $<1$. Solid and dashed curves are from our model with and without GAWBS noise, respectively. Measurements marked by (circles) crosses are (un)corrected for artifacts from the OCR process. 


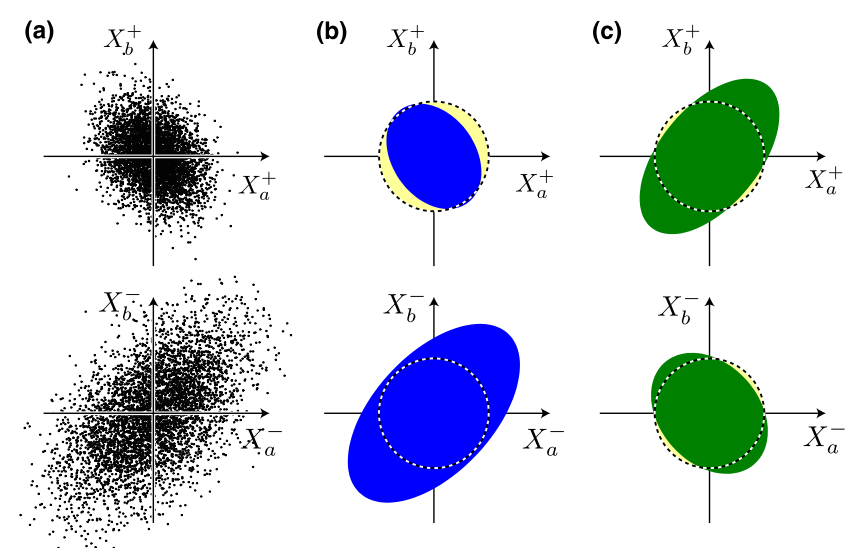

FIG. 4 (color online). (a) Time-series quadrature data (scaled $50 \%$ ). (b) Shaded ellipses follow a contour of the resulting probability distribution. Dashed circles mark the quantum noise limit. The quantum correlation in amplitude is evident since the ellipse falls within the circle. (c) Dual quantum correlations are exhibited by the same data with the correlation matrix brought into standard form.

We completed measurements of the correlation matrix for 11 runs over many days. The ensemble average of those matrix elements in linear scale and their $95 \%$ confidence intervals give

$M_{a b}=\left[\begin{array}{cccc}0.71(1) & 0 & -0.25(1) & -0.02(6) \\ 0 & 2.45(12) & -0.07(10) & +1.42(5) \\ -0.25(1) & -0.07(10) & 0.83(2) & 0 \\ -0.02(6) & +1.42(5) & 0 & 2.56(6)\end{array}\right]$.

The matrix revealed that both colors were amplitude squeezed with $C_{a a}^{++}=0.71(1)$ and $C_{b b}^{++}=0.83(2)$. The phase quadratures showed antisqueezing of $C_{a a}^{--}=$ $2.45(12)$ and $C_{b b}^{--}=2.56(6)$, which imply that the Heisenberg uncertainty relation was satisfied well above the minimum uncertainty bound. These apparent "mixed" state statistics are a requisite of harmonic entanglement. To compute the inseparability, we performed local symplectic transformations $r_{a}=0.11(1)$ and $r_{b}=0.15(2)$ numerically to each mode such that $M_{a b}$ was brought into the standard form. Applying the inseparability definition Eq. (4) returned a value of $I=0.74(1)$, thus confirming the presence of entanglement.

The time-series quadrature data of the second-harmonic field were plotted against the fundamental field in Fig. 4(a). The ellipse in Fig. 4(b) marks the standard deviation contour of the resulting probability distribution. Quantum anticorrelation in amplitude is evident as the ellipse falls within the vacuum states circle. For the phase quadrature, only a classical correlation can be seen, but the proximity of the phase correlations to the classical bound is sufficient for the preservation of entanglement. This feature is symptomatic of biased entanglement $[18,20]$. In Fig. 4(c), we performed local symplectic transformations to bring the correlation matrix into standard form. This led to the amplitude quadratures becoming correlated and the phase quadratures anticorrelated by an equal amount, thereby optimally redistributing the quantum correlations over both quadratures.

In conclusion, we have observed entanglement between two light beams spanning an octave that was generated from an optical parametric amplifier. The results are in good agreement with a theoretical model that was tested across a range of parametric processes. An entanglement source of this type could aid the linking together of two optical systems at different wavelengths, in particular, bridging the octave in optical frequency between telecommunication windows and the prominent multilevel atomic systems used in quantum information processing [22].

We acknowledge support from the Australian Research Council and the Deutsche Forschungsgemeinschaft.

[1] Th. Udem, R. Holzwarth, and T. W. Hänsch, Nature (London) 416, 233 (2002).

[2] R. Holzwarth, et al., Phys. Rev. Lett. 85, 2264 (2000).

[3] M. Fischer et al., Phys. Rev. Lett. 92, 230802 (2004).

[4] E. S. Polzik, J. Carri, and H. J. Kimble, Appl. Phys. B 55, 279 (1992); N. Treps et al., Science 301, 940 (2003).

[5] K. McKenzie et al., Phys. Rev. Lett. 93, 161105 (2004); H. Vahlbruch et al., Phys. Rev. Lett. 97, 011101 (2006); 100, 033602 (2008).

[6] S. A. Diddams et al., Phys. Rev. Lett. 84, 5102 (2000).

[7] S. J. Freedman and J. F. Clauser, Phys. Rev. Lett. 28, 938 (1972); M. Pelton et al., Opt. Express 12, 3573 (2004).

[8] C. Schori, J. L. Sørensen, and E. S. Polzik, Phys. Rev. A 66, 033802 (2002); J. Laurat et al., Opt. Lett. 30, 1177 (2005); A. S. Villar et al., Phys. Rev. Lett. 95, 243603 (2005); X. Su et al., Opt. Lett. 31, 1133 (2006).

[9] M. K. Olsen, Phys. Rev. A 70, 035801 (2004).

[10] A. S. Villar et al., Phys. Rev. Lett. 97, 140504 (2006).

[11] N. B. Grosse et al., Phys. Rev. Lett. 96, 063601 (2006).

[12] Y. Li et al., J. Opt. Soc. Am. B 24, 660 (2007).

[13] M. D. Levenson et al., Phys. Rev. A 32, 1550 (1985); O. Glöckl et al., Opt. Lett. 29, 1936 (2004).

[14] K. N. Cassemiro et al., Opt. Express 15, 18236 (2007).

[15] P. D. Drummond, K. J. McNeil, and D. F. Walls, J. Mod. Opt. 27, 321 (1980).

[16] R. M. Shelby, M. D. Levenson, and P. W. Bayer, Phys. Rev. Lett. 54, 939 (1985); K. Goda et al., Phys. Rev. A 72, 043819 (2005).

[17] M. J. Collett and C. W. Gardiner, Phys. Rev. A 30, 1386 (1984).

[18] L.-M. Duan et al., Phys. Rev. Lett. 84, 2722 (2000).

[19] W. P. Bowen et al., Phys. Rev. A 69, 012304 (2004).

[20] W. P. Bowen, P. K. Lam, and T. C. Ralph, J. Mod. Opt. 50, 801 (2003).

[21] Parameters: $\kappa_{a 1}=51, \kappa_{a 2}=4.3, \kappa_{b 1}=250, \kappa_{b 2}=41$ (in MHz). $\epsilon=1.5 \mathrm{kHz}, \xi_{a}=2.4 \times 10^{-17}$, and $\xi_{b}=$ $3.2 \times 10^{-17}$.

[22] B. Julsgaard et al., Nature (London) 432, 482 (2004); T. Chanelière et al., Nature (London) 438, 833 (2005). 